\title{
A NEW SINGULARITY OF TRANSONIC PLANE FLOWS*
}

\author{
BY \\ A. R. MANWELL \\ University College, Swansea
}

Introduction. The limit line singularity of the hodograph method $[1,2,3]$ cannot arise in transonic plane flows past a physical boundary of finite curvature $[4,5,6,7]$. In this note we discuss a new singularity of the hodograph method which gives infinite acceleration of the fluid but avoids the difficulties of the limit line theory.

1. On the non-occurrence of the limit line in transonic plane flow. We follow Morawetz and Kolodner [7] in regard to notation and write the hodograph equations as

$$
\begin{aligned}
& \varphi_{\theta}=\rho^{-1} q \psi_{a}, \\
& \varphi_{a}=\rho^{-1} q^{-1} c^{-2}\left[q^{2}-c^{2}\right] \psi_{\theta} .
\end{aligned}
$$

Here $\varphi(q, \theta)$ is the potential function, $\psi(q, \theta)$ the stream function, $\rho$ is a known function of $q$ and $c^{2}=d p / d \rho$ where $p=p(\rho)$ is the equation of state.

We define characteristic variables $\alpha$ and $\beta$ by

$$
\begin{aligned}
& 2 d \alpha=\rho q^{-1} k d q-d \theta \equiv d \sigma-d \theta, \\
& 2 d \beta=\rho q^{-1} k d q+d \theta \equiv d \sigma+d \theta,
\end{aligned}
$$

say, where

$$
k^{2}=\rho^{-2} c^{-2}\left(q^{2}-c^{2}\right),
$$

so that $k=k(\alpha+\beta)$, and $k$ increases with $q$.

Then (1.1) and (1.2) may be written

$$
\begin{aligned}
\varphi_{\alpha} & =-k \psi_{\alpha}, \\
\varphi_{\beta} & =k \psi_{\beta} .
\end{aligned}
$$

The limit line is defined by the locus $J=0$, where

$$
J=\frac{\partial(x, y)}{\partial(\theta, q)}=\rho^{-2} q^{-1}\left[\psi_{a}^{2}-k^{2} \rho^{2} q^{-2} \psi_{\theta}^{2}\right]=q^{-3} k^{2} \psi_{\alpha} \psi_{\beta} .
$$

By (1.6) and (1.7), $\psi$ satisfies

$$
2 k \psi_{\alpha \beta}+k_{\alpha}\left(\psi_{\alpha}+\psi_{\beta}\right)=0 .
$$

We shall prove that no limit lines occur if:

(a) the physical boundary has finite curvature, say $K=K(\theta)$, and

(b) the hodograph image of this boundary has a continuously turning tangent which is never in the characteristic direction, say $\beta=\beta(\alpha)$ with $\beta^{\prime}(\alpha)$ finite and nonzero for the equation of the hodograph boundary.

We first show that $\psi_{e}$ and $\psi_{\theta}$, and therefore $J$, are bounded in the supersonic region

${ }^{*}$ Received October 27, 1953. 
and on the sonic line. As in [7] we have

$$
K(\theta)=-(\rho q J)^{-1} \psi_{a} .
$$

Combining Eqs. (1.3), (1.4), (1.8) and (1.10), with $\psi_{\alpha} / \psi_{\beta}=-\beta^{\prime}(\alpha)$ we see that, given $K(\theta)$ and $\beta(\alpha)$, both $\psi_{\alpha}$ and $\psi_{\beta}$ take determinate finite values at each point of the hodograph boundary, i.e. knowledge of the physical boundary and of its hodograph image leads to Cauchy data for the hodograph solution $\psi(\alpha, \beta)$ which is thereby uniquely determined in the supersonic region [6]. Clearly $\psi_{\alpha}$, and $\psi_{\beta}$ are finite at all supersonic points since any singularity would be propagated along at least one characteristic to the boundary where we know that $\psi_{\alpha}, \psi_{\beta}$ are finite. For sonic points we take the origin of $\alpha, \beta$ on the sonic line and note that $k \sim(\alpha+\beta)^{1 / 3}$. (We use the sign $\sim$ to denote the form of the dominant term in an expansion near a singularity and the sign $\cong$ when the numerical factor is significant). The dominant terms in the solutions of Eq. (1.10) must therefore satisfy

$$
6(\alpha+\beta) \psi_{\alpha \beta}+\psi_{\alpha}+\psi_{\beta}=0,
$$

which is homogeneous in $\alpha, \beta$ while the terms neglected are of a higher order. Now $\psi_{\alpha}$, $\psi_{\beta}$ are finite at all supersonic points near $F$, so the theory of Darboux [8, p. 90] applies as $\alpha, \beta$ tend to zero.

In our notation

$$
\psi(\alpha, \beta) \sim(\alpha+\beta)^{2 / 3} g(\alpha, \beta)+h(\alpha, \beta),
$$

where $g$ and $h$ are regular.

We see at once that $\psi_{\theta} \sim \psi_{\beta}-\psi_{\alpha}$ and $\psi_{a} \sim(\alpha+\beta)^{1 / 3}\left(\psi_{\alpha}+\psi_{\beta}\right)$ are bounded on the sonic line. Writing $U$ for the values of $k \psi_{\alpha}$ on the characteristic line $\alpha=$ const., Eq. (1.9) gives

$$
d U / d k=-\psi_{\theta} .
$$

From (1.8) and (1.13) and for values on $\alpha=$ const.

$$
J=-q^{3} k^{2}(d / d k)\left(U^{2} k^{-1}\right) .
$$

If $U$ vanishes for $k=k_{0}$ and $\psi_{\theta}$ is bounded, say $\left|\psi_{\theta}\right|<M$, Eq. (1.13) gives $|U|<$ $M\left(k-k_{0}\right)$ so that $U^{2} k^{-1}$ certainly vanishes at $k=k_{0}$. The case $k_{0}=0$, i.e. where the zero is on the sonic line is included in this argument. Since $U^{2} k^{-1}$ is positive near $k=k_{0}$ we deduce that it is increasing near $k=k_{0}$ and so the derivative in (1.14) is positive. We see then that $J$ changes sign from positive to negative with $k$ and $q$ increasing and a similar argument would show that if $V=k \psi_{\beta}$ vanishes $J$ changes sign along the characteristic $\beta=$ const.

Now conditions (a) and (b) also ensure that $J>0$ on the boundary and the reasoning of Manwell [6] and Morawetz and Kolodner [7] can be applied to complete the proof that $J$ is positive throughout the region.

2. A singularity induced by a corner on the hodograph boundary. We now relax condition (b) of Sec. 1 by admitting a single corner at point $C$ on the hodograph boundary, (see Fig. 1). The solution $\psi(\alpha, \beta)$ is still uniquely defined by giving $\beta=\beta(\alpha)$ and $K=K(\theta)$ but the solution has discontinuities in the first derivatives with respect to $\alpha$ and $\beta$.

Observing that the leading terms in the expansions near $C$ must satisfy $\psi_{\alpha \beta}=0$ we 
find without difficulty that the solutions in regions $I$ and $I I$ may be written

$$
\begin{aligned}
& \psi_{1} \cong d[\alpha(1+\epsilon) /(1-\epsilon)+\beta], \\
& \psi_{2} \cong d\left[\alpha(1+\epsilon) /\left(1+\epsilon^{\prime}\right)+\beta\right],
\end{aligned}
$$

where the tangents at $C$ are $\sigma=\epsilon \theta$ and $\sigma=-\epsilon^{\prime} \theta$ while $d$ is a constant and the origin is at point $C$.

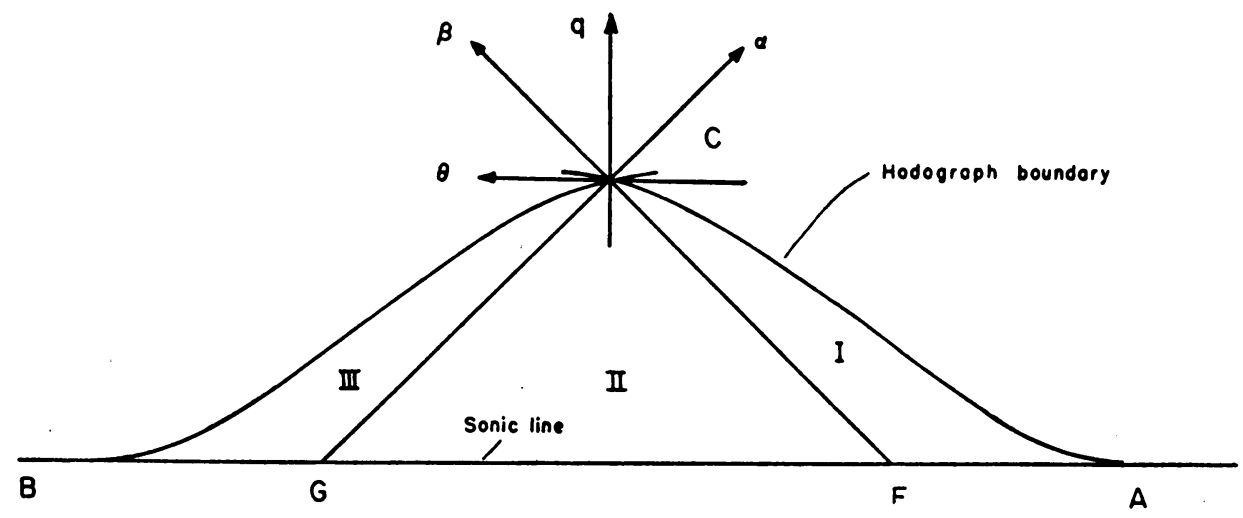

Fig. 1. Hodgraph Plane, Supersonic Region in Characteristic Coordinates $\alpha, \beta$.

The discontinuity in $\psi_{\alpha}$, say $\left[\psi_{\alpha}\right]$, is, see [9, p. 54] and Eq. (1.9) ahove, propagated along the characteristic $\alpha=0$ according to

$$
2 k\left[\psi_{\alpha}\right]_{\beta}+k_{\beta}\left[\psi_{\alpha}\right]=0,
$$

which gives, with $C$ another constant,

Since $\psi_{\beta}$ is continuous at $\alpha=0$

$$
\left[\psi_{\alpha}\right]=C k^{-1 / 2} .
$$

$$
\left[\psi_{\theta}\right]=-\frac{1}{2} C k^{-1 / 2} .
$$

Now the arguments of Sec. 1 still apply to the solution $\psi_{1}$ which is determined by finite data on the smooth curve $A C$ independently of how $A C$ is continued beyond $C$. In particular $\left(\psi_{1}\right)_{\theta},\left(\psi_{1}\right)_{a}$ and $J_{1}$ are finite along $C F$. Using Eqs. (2.4), (2.5) and (1.8) we find without difficulty that, at $F,\left(\psi_{1}\right)_{a}=\left(\psi_{2}\right)_{a}$ and $J_{2}=J_{1}$, which is non-zero according to Sec. 1, but $\left(\psi_{2}\right)_{\theta}$ tends to infinity near $F$ with $k^{-1 / 2}$. According to Eq. (1.10) the streamline curvature remains finite at $F$ but for the fluid acceleration, say $f$, we have $f_{2}=q$ $d q / d s=q(d q / d \theta)_{\psi=\text { const. }} .(d \theta / d s)=-\left(q K \psi_{\theta} / \psi_{\theta}\right)_{2}$ which tends to infinity with $\psi_{\theta}$.

In the limit line singularity both $K$ and $f$ become infinite together, a difficulty which is avoided here.

3. Expansion of the solution near the singularity. We write $[\psi]$ for $\psi_{2}-\psi_{1,(2)}$ and set $[\psi]=0$ in region $I$ while $\psi_{1,(2)}$ may be any flow past a smooth profile and determined in region $I I$ by data on $A C$ and $A C$ slightly produced beyond $C$ with no discontinuity in slope at $C$. Taking the origin at $F$ we construct the leading term in the expansion of a solution of Eq. (1.9) as a homogeneous function of $\alpha$ and $\beta$ satisfying Eq. (1.11), viz.,

$$
[\psi] \sim \alpha \beta^{-1 / 6} F\left(1 / 6,7 / 6,2,-\alpha \beta^{-1}\right)
$$


for $0 \leq-\alpha \beta^{-1} \leq 1$ and with $F(a, b, c, z)$ denoting the hypergeometric series (see [10, Chap. 10]).

For $\alpha=0$ we have $[\psi] \equiv 0$ and, in conformity with Eq. (2.4),

$$
\left[\psi_{\alpha}\right] \sim \beta^{-1 / 6} \sim k^{-1 / 2} .
$$

Now $[\psi]$ is the only homogeneous solution satisfying this requirement and regular in $0 \leq-\alpha \beta^{-1} \leq 1$. For $\left|\alpha \beta^{-1}\right|<1$ Eq. (3.1) may be differentiated term by term and we find that $[\psi]_{a} \sim \theta^{1 / 6}$ and $[\psi]_{\theta} \sim \theta^{-1 / 6}$. For almost all points in the hodograph plane near $F, z=-\alpha \beta^{-1}$ is nearly unity and we use the identity given by Copson [10, p. 252] yielding

$$
\begin{aligned}
{[\psi] \sim \alpha \beta^{-1 / 6}[P F(1 / 6,7 / 6,1 / 3,1} & \left.+\alpha \beta^{-1}\right) \\
& \left.+Q\left(1+\alpha \beta^{-1}\right)^{2 / 3} F\left(11 / 6,5 / 6,5 / 3.1+\alpha \beta^{-1}\right)\right],
\end{aligned}
$$

where $P$ and $Q$ are certain numerical constants.

Writing $q=c_{*}+\lambda t$ we choose the constant $\lambda$ so that $\sigma=t^{3 / 2}$ and for all finite values of $t / \theta$

$$
[\psi] \cong \theta^{5 / 8}\left[P+2^{2 / 3} Q t \theta^{-2 / 3}\right] .
$$

We can show too that Eq. (3.4) may be differentiated giving

$$
\begin{aligned}
& {[\psi]_{\theta}=(5 / 6) P \theta^{-1 / 6},} \\
& {[\psi]_{\imath}=2^{2 / 3} Q \theta^{1 / 6} .}
\end{aligned}
$$

From these results for the derivatives of $[\psi]$ it follows that $J_{2}-J_{1,(2)}$ is uniformly small and that in spite of the infinity in acceleration at $F$ the physical coordinates near $F$ are changed very little by adding $[\psi]$ to $\psi_{1,(2)}$. Although there is a discontinuity in $\psi_{\alpha}$ along $F C$ the physical solutions join up smoothly because, according to (1.6) and (1.7), not only $\psi$ but $\varphi$ is continuous between $I$ and $I I$.

4. The continuation of the solution across the sonic line. In general, of course, we may not joint up piecewise differentiable hodograph solutions, for the corresponding physical planes would not join up. In particular we require continuity of both $\psi$ and $\psi_{\text {a }}$ at the sonic line (see Fig. 2). We write

$$
\theta=s \cos \theta, \quad|t|^{3 / 2}=s \sin \theta,
$$

with $0 \leq \theta \leq \pi$. Suitable solutions are found as the real and imaginary parts of

$$
\Psi \sim s^{5 / 8} \exp [7 i(\pi-\theta) / 6] F(1 / 6,7 / 6,2, \exp [-2 i \theta]),
$$

and two further solutions are found by replacing $F$ by

$$
G=F \int Z^{-2} F^{-2}(1-Z)^{-1 / 3} d Z
$$

where $Z=\exp (-2 i \theta)$.

Except in the vicinity of $\theta=0$ we have either $\theta$ nearly zero or $\theta$ nearly $\pi$ and so $Z$ is nearly unity in either case. Using Eq. (3.3) for complex values of $Z$ and Eqs. (4.2), (4.3) it is not difficult to show that, except for $\theta$ nearly zero, the most general complex $\Psi$ is given by

$$
\Psi \sim \theta^{5 / 8} \exp [7 i(\pi-\theta) / 6]\left[R+S|\sin \theta|^{2 / 3} \exp [i(\pi-2 \theta) / 3],\right.
$$


where $R$ and $S$ are arbitrary constants.

Taking $S$ zero we find a solution

$$
\Psi_{1} \cong \theta^{5 / 6} \sin [7(\pi-\theta) / 6],
$$

and similarly making $R$ zero we get

$$
\Psi_{2}=\theta^{1 / 6}|t| \sin [11(\pi-\theta) / 6] .
$$

Once more it can be shown that these expressions may be differentiated with respect to $t$ or $\theta$ and easily $d \theta / d t=0$ for $t=0$ so both derivatives vanish on $\theta=\pi$, i.e. $\Psi_{0}=$ $\Psi_{a}=0$ for $\theta<0$. We form the combination

$$
[\Psi]=2 P \Psi_{1}+2^{5 / 3} Q \Psi_{2}
$$

and verify that it satisfies both $\Psi=\Psi_{a}=0$ for $|t|=0$ and $\theta<0$ and conditions (3.5) and (3.6) for $|t|=0$ and $\theta>0$.

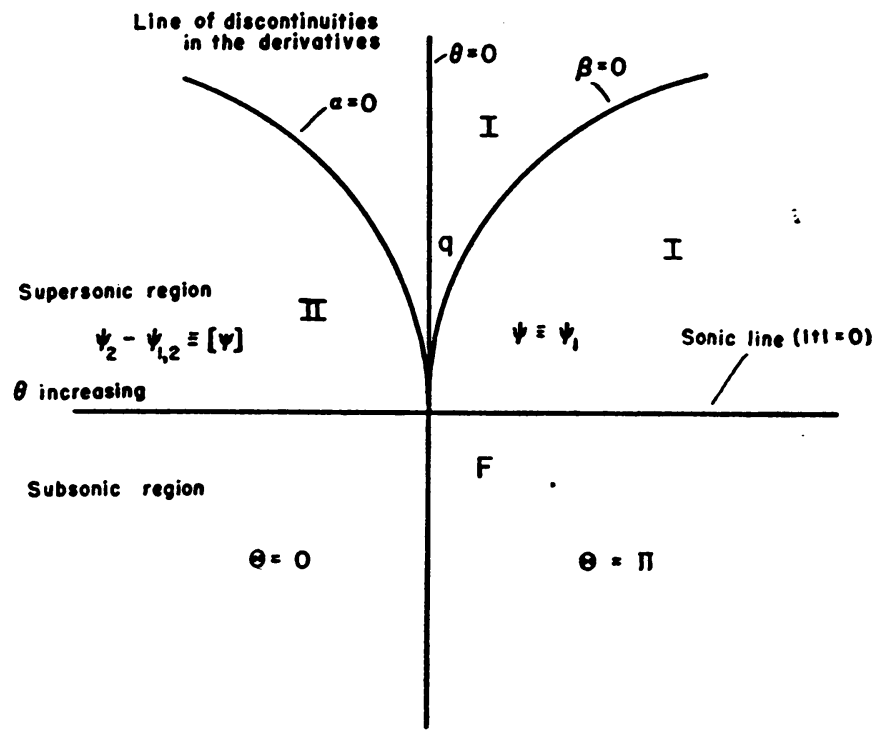

FIg. 2. Hodograph Plane Near Critical Point F.

A simple discussion shows that the other two linearly independent solutions given by Eq. (4.4) cannot occur and so [ $\Psi]$ which was uniquely determined in region II, save for a constant multiplier depending on data near $C$, has a unique continuation across the sonic line, viz. [ $\Psi$ ] of Eq. (4.7). It is easily verified that for any $\Psi$ and near $\theta=0$ we have $\Psi_{\theta} \sim|t|^{-1 / 4}$ and $\Psi_{t} \sim|t|^{-1 / 4}$. (We use Eqs. (4.2) and (4.3) and prove that $F(Z)$ has no roots on $|Z|=1$ ). Noting also Eqs. (4.5) and (4.6) we see that $\Psi$ has finite derivatives at all subsonic points near $F$. The solution in the physical plane can be constructed by adding $[\Psi]$ to the continuation across $|t|=0$ of the function $\psi_{1}$ and performing the usual integrations.

The discussion of Secs. 2, 3, 4 applies with obvious changes to the propagation of discontinuities along the other characteristic $C G$ and a singularity develops similarly at $G$ (Fig. 1). 
5. The occurrence of the singularity in flow past a given profile. According to Sec. 1, transonic flows can be constructed in the neighborhood of an arbitrary convex physical boundary of finite curvature along which the velocity is determined by a smooth hodograph image. By Sec. 2, however, there are infinitely many adjacent velocity distributions for which the flow must break down at the sonic line, viz. those for which the hodograph images have any discontinuity in slope at supersonic points.

We turn now to the possibility that the singularity described above may arise for a given body in a subsonic stream as the Mach number of the stream is slowly increased. It must be admitted that the existence and uniqueness of plane transonic flow solutions in this situation is by no means certain. As for uniqueness, the author has proved [11] that the solutions for cyclic flow round a circular cylinder are not unique at an enumerable infinity of values of the circulation parameter and that these values lie close together. For non-circulatory flows past a precisely defined body it is possible that existence of the solutions will fail at certain (perhaps 'almost all') Mach numbers with transonic flow conditions (c.f. Frankl [12], although the argument of [12] is by no means complete).

On the other hand in a number of approximations worked out by the present writer it seems clear that the general consequence of increasing the strength of the stream for an approximately determined body is to give velocity distributions whose hodograph boundaries develop a corner at the point of maximum velocity in the supersonic region.

An example of this process is furnished by [13] which although, as appears on closer examination, merely an approximate solution of the problem implied by the title is a useful contribution to the problem of the behaviour of transonic flow when the boundary is kept fixed while the stream conditions are varied. (The solution of [13] satisfies correct boundary conditions on the arc $B D$ and on the axis $A B$, (Fig. 3) and the flow becomes

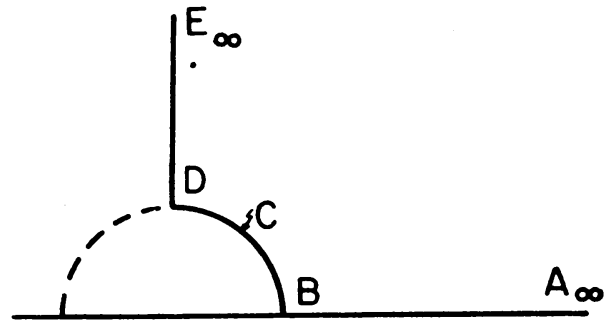

Fig. 3.

uniform at great distances but, owing to lack of analytic continuation the velocity vector along $D E$ need not be parallel to $A B$ except at $D$ and $E$.)

According to [13] the image of the boundary is determined in the hodograph plane by an equation of the form

$$
\cos \theta=\lambda(q) / \lambda\left(q_{1}\right),
$$

where $q_{1}$ is the maximum velocity on the boundary. If $q_{1}<q_{2}$, where $\lambda^{\prime}\left(q_{2}\right)=0$, this equation may be expanded as

$$
q-q_{1} \cong-\frac{1}{2}\left[\lambda^{\prime}\left(q_{1}\right)\right]^{-1} \lambda\left(q_{1}\right) \theta^{2}
$$

If however $q_{1}=q_{2}$ the expansion must be written as

$$
\lambda^{\prime \prime}\left(q_{2}\right)\left(q-q_{2}\right)^{2}=-\lambda\left(q_{2}\right) \theta^{2} .
$$


For the semi-circular body which has in fact been treated in [13] it was found that

$$
\lambda \cong q / c-(5 / 24)(q / c)^{3}-[125 / 3456+35(\gamma-1) / 576](q / c)^{5},
$$

where the notation is that of Courant and Friedrichs [9]. To this approximation it appears that $\lambda^{\prime}(q)$ vanishes for $q_{2} \cong 1.03 c$ and that the branching streamlines in the hodograph plane make an angle arc $\tan (q d \theta / d q)=1.86$ with the line $\theta=0$.

In this example the singularity develops for only a very small supersonic region but in other cases of transonic flow worked out by the author to the same degree of approximation the supersonic region appears to develop considerably before the flow breaks down.

\section{REFERENCES}

1. G. I. Taylor, Recent work on the flow of compressible fuids, I Lond Math. Soc. 5, 224 (1930).

2. Th. von Kármán, Compressibility effects in aerodynamics, J. Aero. Sci. 8, 337-356 (1941).

3. H. S. Tsien, The limiting line in mixed subsonic and supersonic flow of compressible fluids, T.N. 961, N.A.C.A. (1944).

4. A. A. Nikolskii and G. I. Taganov, Gas motion in a local supersonic region and conditions of potential flow breakdown, transl. from Prikl. Mat. Mekh. 10, 481 (1946) by S. Reiss, N.A.C.A. Tech. Mem. 1213.

5. K. O. Friedrichs and D. Flanders, On the non-occurrence of a limiting line in transonic flow, Com. Appl. Math. 1, 287-301 (1948)

6. A. R. Manwell, A note on the hodograph transformation, Quart. Appl. Math. 10, 177-184 (1952).

7. C. S. Morawetz and I. I. Kolodner, On the non-existence of limiting lines in transonic flows, Com. Pure Appl. Math. 6, 97-102 (1953).

8. G. Darboux, Leçons sur la théorie générale des surfaces, vol. 2, Paris, 1915.

9. R. Courant and K. O. Friedrichs, Supersonic flow and shock waves, Interscience Publishers, Inc., New York, 1948.

10. E. T. Copson, Theory of function of a complex variable, Oxford, 1935.

11. A. R. Manwell, The variation of compressible flows, Quart. J. Mech. Appl. Math. 7, 40-50 (1954).

12. F. I. Frankl, On the formation of shock waves in subsonic flows with local supersonic velocities, transl. N.A.C.A., T.M. 1251.

13. A. R. Manwell, Expansion in series of the exact solution for compressible flow past a circular or an elliptic cylinder, Phil. Mag. (7) 36, 499-510 (1945). 